\title{
Chaotic Genetic-fuzzy Encryption Technique
}

\author{
Hamdy M. Mousa \\ Faculty of Computers and Information, Menoufia University, Egypt \\ E-mail: hamdimmm@hotmail.com
}

Received: 19 November 2017; Accepted: 16 January 2018; Published: 08 April 2018

\begin{abstract}
As the result of increasing use of internet in daily communication and the importance of information security during data storage and transmission process, we propose iterative Chaotic Genetic-fuzzy Encryption Technique(C-GET) in order to enhance secured encryption technique and less predictable. In this technique,binarize any digital data type. The main encryption stages of C-GET are chaotic map functions, fuzzy logic and genetic operations. Mathematic operations and rotation are also included that increase encryption quality. Images are used for testing propose. For testing C-GET,digitalimagesareusedbecause they become an important resource of communication. The original and reconstructed data are identical. Experimental results show that C-GET technique has multilayer protection stages against various attacks and a powerful security based on the multi-stages, multiple parameters, fuzzy logic and genetic operations. Decrypted data is nearly randomness and has negligible correlation with secret data.
\end{abstract}

Index Terms - Cryptography, chaotic function, fuzzy logic and Genetic Algorithm.

\section{INTRODUCTION}

Nowadays, data such as digital images, audio, video becomes a vital resource of communication. There are many intruders and attackers try to unauthorized access sensitive data. Therefore, the need to build robust and efficient security techniques for protection of digital data during transmission or storage them over the internet is vital demand [1-2]. A possible traditional solution is a cryptography. It is the science to encrypt and decrypt data to transmit or store sensitive information over insecure networks so that only the intended recipient can read it. Encryption technique type may be symmetric that uses only one secret key for encryption and decryption or asymmetric that uses one secret key for encryption and another key for decryption [3]. The key length and operation's functions are important characteristics that determine the strength and efficiency of encryption technique. A strongencryption technique must verify confusion and diffusion requirements and resistant to cryptographic attacks. Thetraditional encryption techniques like DES, AES, IDEA, RSA, etc. are used and gave good results [1- 5]. In [6], Symmetric Key Encryption Decryption using modulo 69 is proposed for encryption and decryption process.

Using traditional encrypting techniques in real-time multimedia applications suffers from high computing power, large computation time and high expenses. Hence, it demands bettersolutions to resolve the security problems of multimedia data effectively [7].The authors described an approach for high-quality image hiding by improving the visual quality of the stego-image with large embedding capacity [8]. In [9], Omar A. Dawood and et.al., proposed Tigris cipher based on secret-key block cipher to protect privacy and resist attacks. This cipher applies essentially four functions to produces an adequate security with minimum encryption time. These functions are XORed, S-box tables, row shifting and mixing each column in the matrix operation.

In [10], an extension of Hill cipher by generating dynamic encryption key matrix is proposed to provide more effective in the encryption quality of RGB images.

Authors Proposed encryption algorithm for enhancing the security level of the encrypted image that comprises two phases: fuzzy logic system and genetic algorithm that generate key value [11]. Authors proposed ascheme to enhance securitylevelfor image encryption based on thesecret sharing concept, fuzzy logic technique and AES algorithm [12].

Paper [13] proposed an algorithm to generate server public and private keys using fuzzy logic for decreasing encryption and decryption processing time. The author proposed a modified pixel-chaotic-shuffle mechanism for color image encryption based on multi-chaotic systems for improving security level [14]. The authors proposed encryption and decryption methodology for enhancing the security by adding layer of security to encode of characters that is performed based on magic rectangle. This methodology is acting as a wrapper to any public key cryptosystems for enhanced security [15]. For enhancing image encryption, the algorithm by dividing it into a number of blocks based on the Fuzzy sets is proposed [16]. In [17], a technique-basedon Fuzzy approach, variable multiple rights translated AES Gray Sboxes and steganography technique for gray image encryptionis presented that has high resistanceagainst computational attacks. An iterative symmetric encryption algorithm based on a combination of bit permutations andchaotic function to enhance digital data securityis presented in [18].

The paper evaluates and analyzes image encryption techniques that are proposed in the literature and verify their characteristics to determine the Strength, efficiency 
and weaknesses of these encryption techniques [19].

The authors introduced DNA Encryption and Decryption Algorithmby generating a DNA encoding table for encoding of secret data, division, rotation and transformation [20-21].

In [22], the author proposed DNA-Genetic Encryption Technique based on the multi-stage and genetic operations torealize a great confidentiality system.

Many cryptosystems implement simultaneously confusion and diffusion stages for strong encryption effectand high security. Image encryption algorithms using the chaotic map to shuffles the pixel positions and modifies pixel values in plain-image to get unrecognizable image [23]. Fridrich, J. [24] proposedencryption algorithm to image using twoDimension chaotic map. Design image cryptosystems using three-Dimension chaotic map to improve encryption efficiency anddecreases processing time are proposed in [25-28].

This paper proposes Chaotic Genetic-fuzzy Encryption Technique(C-GET) that is an iterative symmetric technique to encrypt a secret data. C-GET depends on standard chaotic, chaotic Henon map, some of the mathematic operators, fuzzy logic system and genetic algorithm operations. C-GET composes of pre-processing symmetric key encryption and genetic-fuzzy operation encryption stage. In this technique, the secret data converted into binary. In addition, generate chaos functions values related to predefined initial values then create appropriate fuzzy members and fuzzy rules based on experience. Each round has multiple operations dependent on chaotic map values. In addition to that, the symmetric key is used. Anydata type format can be used as secret data i.e. text, word document, image pixels, audioandvideo. The color and gray images offer an excellent digital data to test any encryption technique so; they are used for testing the efficiency of the proposed technique. Experimental results prove that typical copy of secret data is reconstructed. They also show that proposed technique improves encryption efficiency. The remaining of this paper is organized as follows: the proposed technique is introduced in detail in section 2; the experimental results are presented, discussed and evaluated in section 3 . The obtained security results are satisfied. Finally, the last section gives concluding remarks.

\section{ChaOtic GenetiC-FuZZY ENCRYPTION TECHNIQUE}

The main objective of all security techniques is to protect information and data from any attacker activities. Due to increasing of computing power, time and computational complexity are two significant parameters those making security algorithms strong and unbreakable. The symmetric cryptography technique is an iterative process, which is proposed to encrypt a secret data. The proposed technique is depended on the standard chaotic function, chaotic Henon map, some of the mathematic operators, fuzzy logic system and genetic algorithm operations. Any digital data type (i.e. message, signal, image or video) can be encrypted. The main steps of the proposed technique are pre-processing, symmetric key encryption, and genetic-fuzzy operation encryption stage. They are explained as follows.

\section{A. Pre-processing Stage}

After reading secret data, this data must be prepareddependingon its type. In case of atext file, it is converted into ASCII values. Group them into 8-bits binary data. In case of a gray image, read pixels of image data into 8-bits Binary data. In RGB image, first, it is separated into three components. As it is known, a classic video includes many frames. Separate one frame at a time. The same steps of the gray image is applied for every RGB components and each video frames. After grouping secret data (message, image, video or signal) into bytes, convert it into two-dimension (2D) matrix.

\section{B. Encryption Stage}

The original input data is treated as a simple 2D matrix composed of bits " 0 " and " 1 ". After read the secret data, encrypt it using a standard chaotic function given by:

$$
f(x)=\lambda x(a-x)
$$

With $\lambda$ value between 3.57 and 4 [29] and $a$ is approximately equal one. The chaotic behavior of such a function has been widely studied. The chaotic system is sensitive to a very slight change in initial condition or parameters.

Generate chaotic random values equal the size of 2D matrix then, perform an exclusive OR operation on the corresponding elements of them.

\section{Genetic-fuzzy Operation Encryption Stage}

Another chaotic function is used to define the number of iterative process, positions' index and the type of operations that are executed. This is a two-dimensional chaotic map that is called a Henon map (HM) and its equations are:

$$
\begin{gathered}
x(n+1)=y(n)-b x^{2}(n)+1 \\
y(n+1)=c x(n)
\end{gathered}
$$

When using this map, initial values and the parametersmust be set, the system behaves as a chaotic system when $b=1.4$ and $c=0.3$ [30].

This chaotic system generates a chaotic sequence of real random numbers. These real numbers are transformed to a sequence of unsigned random numbers byusing the following equation:

$$
\begin{gathered}
x(n)=x(n)+\max (-x(n))) \\
x(n)=\operatorname{round}(d *(x(n))
\end{gathered}
$$

Conversion unsigned random numbers $(x)$ to the approximate size of $2 \mathrm{D}$ matrix that represents encrypted secret data with suitable $d$ value. 
In general, the encryption system consists of two stages: replacement of datavalues called confusion and modification of data values called diffusion. To execute confusion and diffusion, the proposed system defines a number of operations based on the mathematic operator, fuzzy logic and genetic algorithm stages.

It is known that fuzzy concepts first introduced by Zadeh in the 1960s and 70s and Fuzzy logic attempts to reflect the human way of thinking. In a global sense, fuzzy logic is approximately identical with the fuzzy sets theorem, which relates to classes of objects with un-sharp boundaries in which membership is a matter of degree [31-32]. Types of Fuzzy algorithms arerelational algorithms that describe a relation between fuzzy variables and can be used to approximately describe behavior of a system and decisional algorithms type which describe a strategy for performing some task. Fuzzy logic algorithm composes from the following steps: fuzzify step that converts input values into fuzzy membership functions, compute the fuzzy output by executing appropriate rules in the rule base and defuzzify that estimates crisp output values.

In the first step of the fuzzy algorithm, divide the twodimension secret data into a number of classes, which represent Fuzzy sets. Define the linguistic variables of fuzzy sets that are sharp or un-sharp boundaries. There are many different membership functions such as triangular, trapezoidal, piecewise linear, Sigmoid, Gaussian, Z-shaped curve, Pi-shaped curve, S-shaped curve or singleton. As an example, figure 1 shows the membership functions.

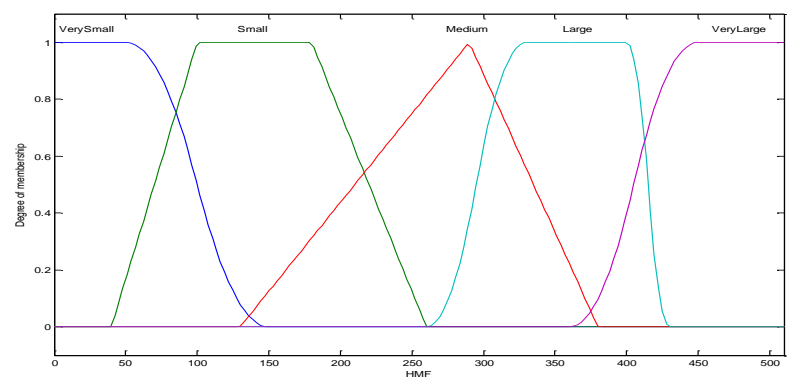

Fig.1. Membership Functions

Before construction the rule base, a number of functions based on mathematics, genetic algorithm (GA) and fuzzy logic operators. The GA includes the following operations: selection, crossover (single point ...) and mutation [33]. These operations are functions that representing the individual transformations. Two random values are generated based on Henon mapping and other predefined values. The first random value is calledposition index that defined that start point of operation. The second random value $(n)$ defines the number of operation's reiteration.

$$
\begin{gathered}
\text { position }=\operatorname{modulus}(x(n), \text { predefined value }) \\
n=\operatorname{modulus}(x(n), \text { predefinedvalue })
\end{gathered}
$$

Selection position must cover all data. The position value selects data for performing some task. A number of functions; that may use in the proposed system; are explained in the following:

- Rotate up $(\operatorname{rot} U(i 1, p o s+1, n))$ : rotate $2 \mathrm{D}$ secret data's row up from row index(position) with $\mathrm{n}$ rows.

- Rotate bottom $(\operatorname{rotB}(i 1, p o s+1, n))$ : rotate $2 \mathrm{D}$ secret data's row bottom from row index with $\mathrm{n}$ rows.

- Rotate right $(\operatorname{rotR}(i 1, p o s+1, n))$ : rotate $2 \mathrm{D}$ secret data's Column right from Column index with $n$ Columns.

- Rotate left $(\operatorname{rotL}(i 1, p o s+1, n))$ : rotate $2 \mathrm{D}$ secret data's Column left from Column position with $n$ Columns.

- Row Complement $(\operatorname{compR}(i 1, p o s+1, n))$ : bit complement 2D secret data's row value from row position until row index equal the addition of position and $n$ value.

- Column Complement $(\operatorname{compC}(i 1, p o s+1, n))$ : bit complement 2D secret data's Column pixel from Column position until Column index is (position + n) value.

- Column Crossover $(\operatorname{cross} C(i 1, p o s+1, n))$ : two Columns (position and position +1) are selected. The position is a single-point crossover point is determined then, exchanging the heads of two Columns. In next step, repeat the following n-times, increase column number with one, select this column and next column and swap two heads of columns at position index.

- Row Crossover (crossR(il,pos $+1, n))$ : two rows (position and position +1 ) are selected. Determine a single-point crossover point position then, exchanging the heads of two rows. In next step, repeat the following $n$-times, increase row number with one, select this row and next row and swap two heads of rows at position index.

- Row mutation (mutateR(il,pos $+1, n))$ : change values (position) with value of any linear function. Repeat this operation n-time.

- Column mutation (mutateC(i1,pos+1,n)):repeat change values (position) value of any linear function for n-times.

- Row addition $(\operatorname{addR}((i 1$, pos $+1, n))$ : add two rows (position and position +1 ). In next step, repeat the following n-times, increase row number with one, add two rows (position and position +1 ).

$$
\begin{aligned}
& \operatorname{Row}(\text { pos })=(\operatorname{double}(\operatorname{Row}(\text { pos }))+ \\
& \quad \text { double }(\operatorname{Row}(\text { pos }+1))>255) *(-255)+ \\
& (\text { double }(\operatorname{Row}(\text { pos }))+\operatorname{double}(\operatorname{Row}(\text { pos }+1)))
\end{aligned}
$$

- Column addition $(\operatorname{add} C((i 1, p o s+1, n))$ : add two Columns (position and position +1 ). In next step, repeat the following n-times, increase Column index with one, add two Columns (position and position +1 ) as the following equation:

\section{Column $($ pos $)=(\operatorname{double}(\operatorname{Column}($ pos $))+\operatorname{double}(\mathrm{Col}$} umn $(\operatorname{pos}+1))>255) *(-255)$ 
$+(\operatorname{double}(\operatorname{Column}($ pos $))+\operatorname{double}(\operatorname{Column}(\operatorname{pos}+1)))$

- Row subtraction $(\operatorname{subR}((i 1, p o s+1, n))$ : subtract row(position+1) from row(position). In next step, repeat the following n-times, increase row index with one, subtract row (position+1) from row (position).

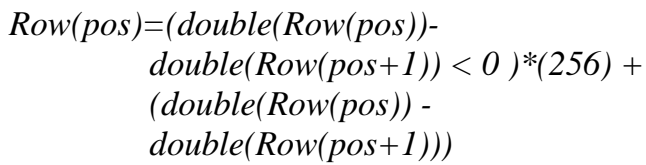

Or subtract row(position) from row(position+1)

- Column subtraction $(\operatorname{subR}((i 1, p o s+1, n))$ : subtract Column(position+1) from Column(position). In next step, repeat the following n-times, increase row index with one, subtract Column(position+1) from Column(position).

\section{$\operatorname{Column}($ pos $)=(\operatorname{double}(\operatorname{Column}($ pos $))-$ double $($ Column $($ pos +1$))<0) *(256)+$ (double(Column(pos))- double $($ Column $($ pos +1$)))$}

It is also used other operation that enhancing encryption process i.e. row/column subtraction from or addition to pseudo-random sequences, chaotic Henon mapping values.

\section{Fuzzy Rules Construction}

Expert knowledge is made decisions as group of if.....then.... statements. Many necessary rules can be written to describe the system adequately and to cover all data. The Fuzzy rules are built dependent on Henon mapping and other predefined values. The condition may be modulus or other operation of Henon mapping values with boundaries Fuzzy sets. Then determine position and $n$ values and apply one or more pre-defined functions, i.e. rotate right, Row subtraction, Column Complement. The rule may have single condition or two conditions. The sample of rules as follows:

if $\operatorname{modulus}(x(i), v a l) \quad>=115 \quad \& \& \mathrm{HMF}$ input is VerySmall;

then determine position and $n$ values and apply $\operatorname{rot} U(i 1, p o s+1, n) ; \operatorname{compR}(i 1, p o s+1, n) ; \operatorname{cross} C(i 1, p o s$ $+1, n)$;

if modulus $(x(i), v a l)>=105 \& \&$ HMF input is Small; then determine position and $n$ values and apply $\operatorname{rot} B(i 1, p o s+1, n) ; \operatorname{comp} C(i 1, p o s+1, n)$;

if modulus $(x(i), v a l)>=90 \& \&$ HMF input is Medium; then determine position and $n$ values and apply $\operatorname{rotR}(i 1, p o s+1, n) ; \operatorname{comp} C(i 1, p o s+1, n)$; if modulus $(x(i), v a l)>=70 \& \& \mathrm{HMF}$ input is Large; then determine position and $n$ values and apply $\operatorname{rotL}(i 1, p o s+1, n) ; \operatorname{compR}(i 1, p o s+1, n)$; if $\operatorname{modulus}(x(i), v a l)>=35 \& \&$ HMF input is VeryLarge; then determine position and $n$ values and apply $\operatorname{rotB}(i 1, p o s+1, n) ; \operatorname{cross} C(i 1, p o s+1, n)$;

if $\operatorname{modulus}(x(i), v a l)>=0$;

then determine position and $n$ values and apply $\operatorname{rot} U(i 1, p o s+1, n) ; \operatorname{rot} L(i 1, p o s+1, n)$; $\operatorname{cross} R(i 1, \operatorname{pos}+1, n)$;

Execution of this process simultaneously mixes the properties of confusion and diffusion that are required to obtain a high-security level. The stages of C-GET are illustrated in figure 2 .

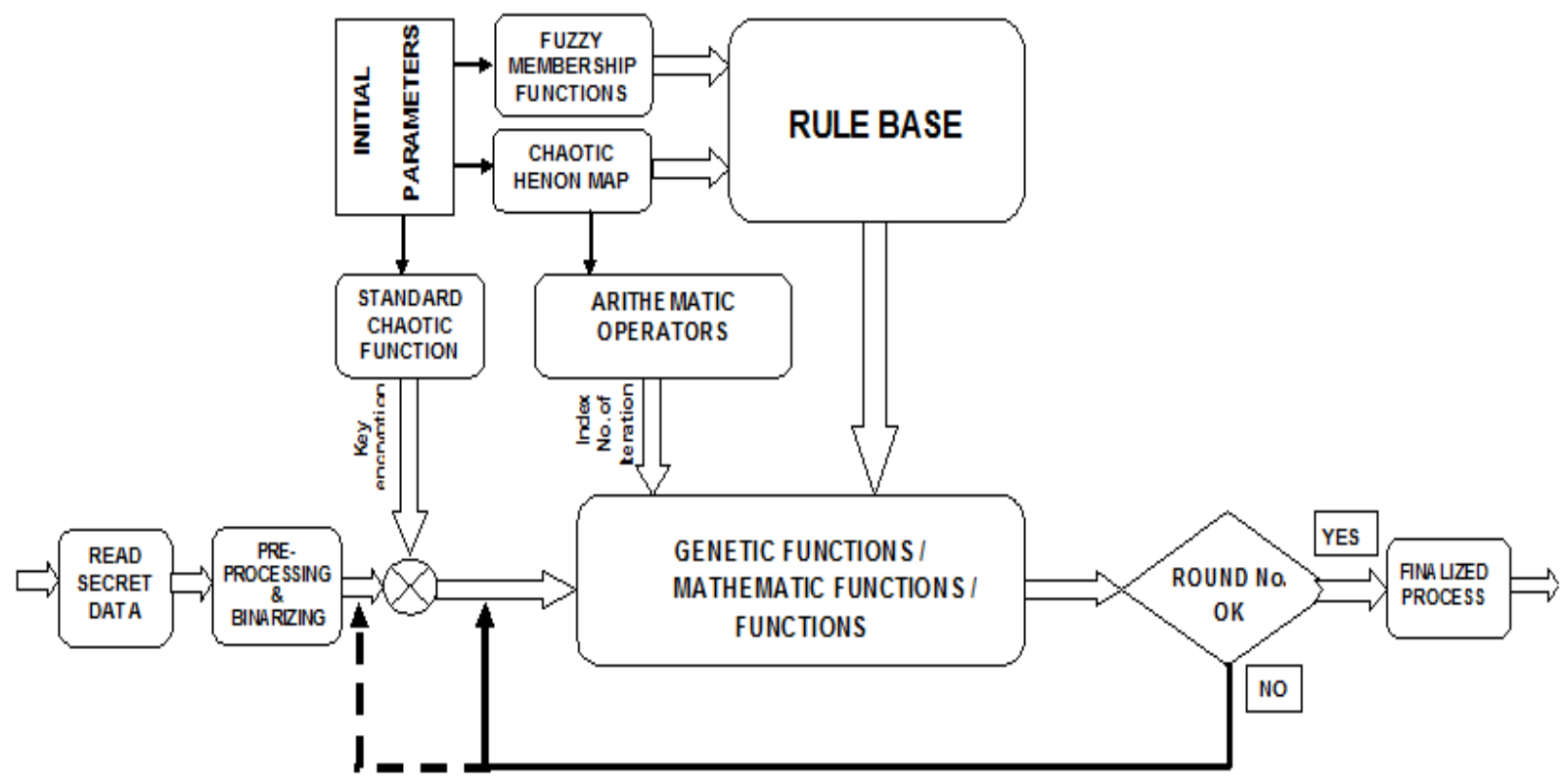

Fig.2. Stages of C-GET 


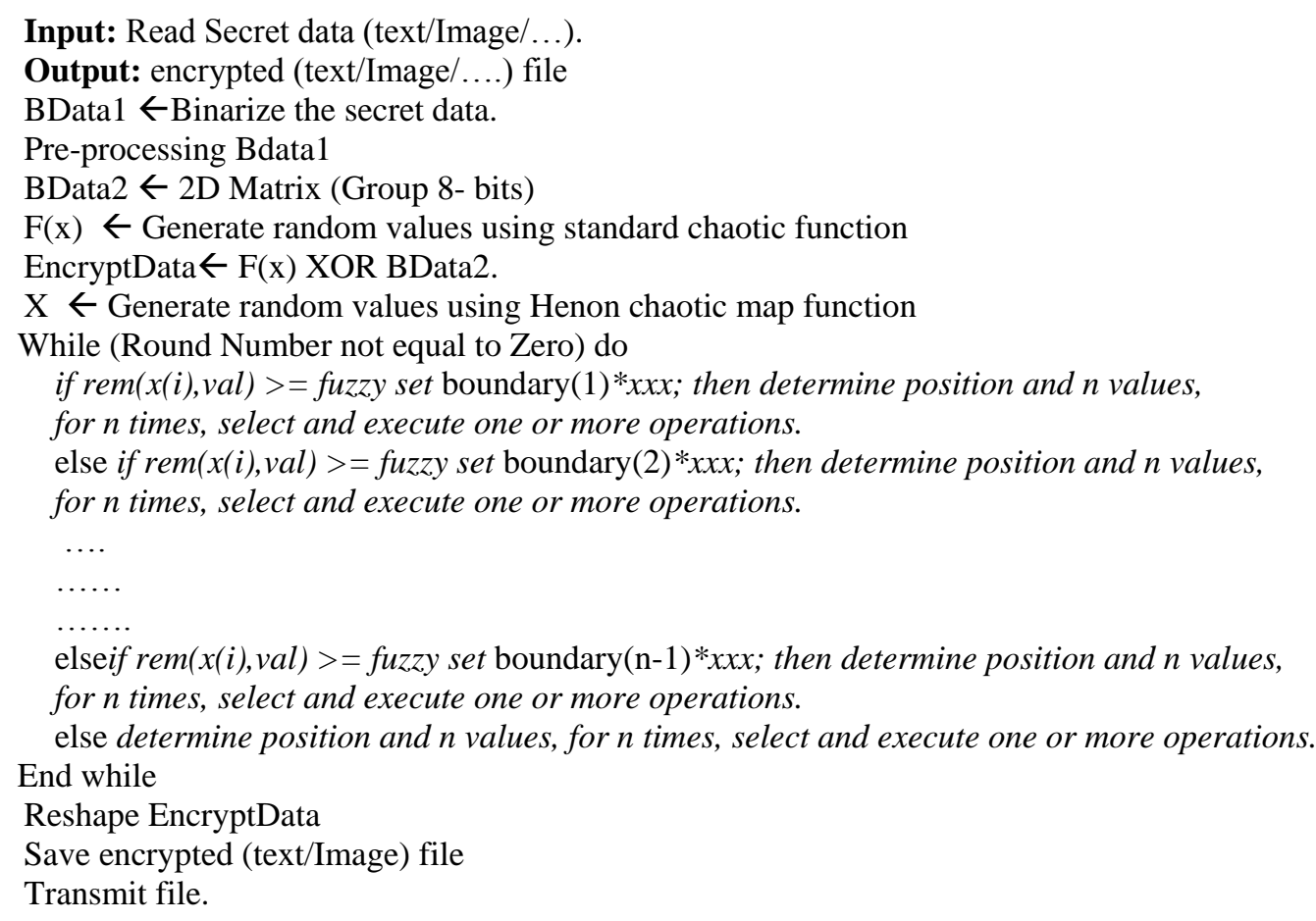

Fig.3. Pseudocode of Proposed Encoding C-GET

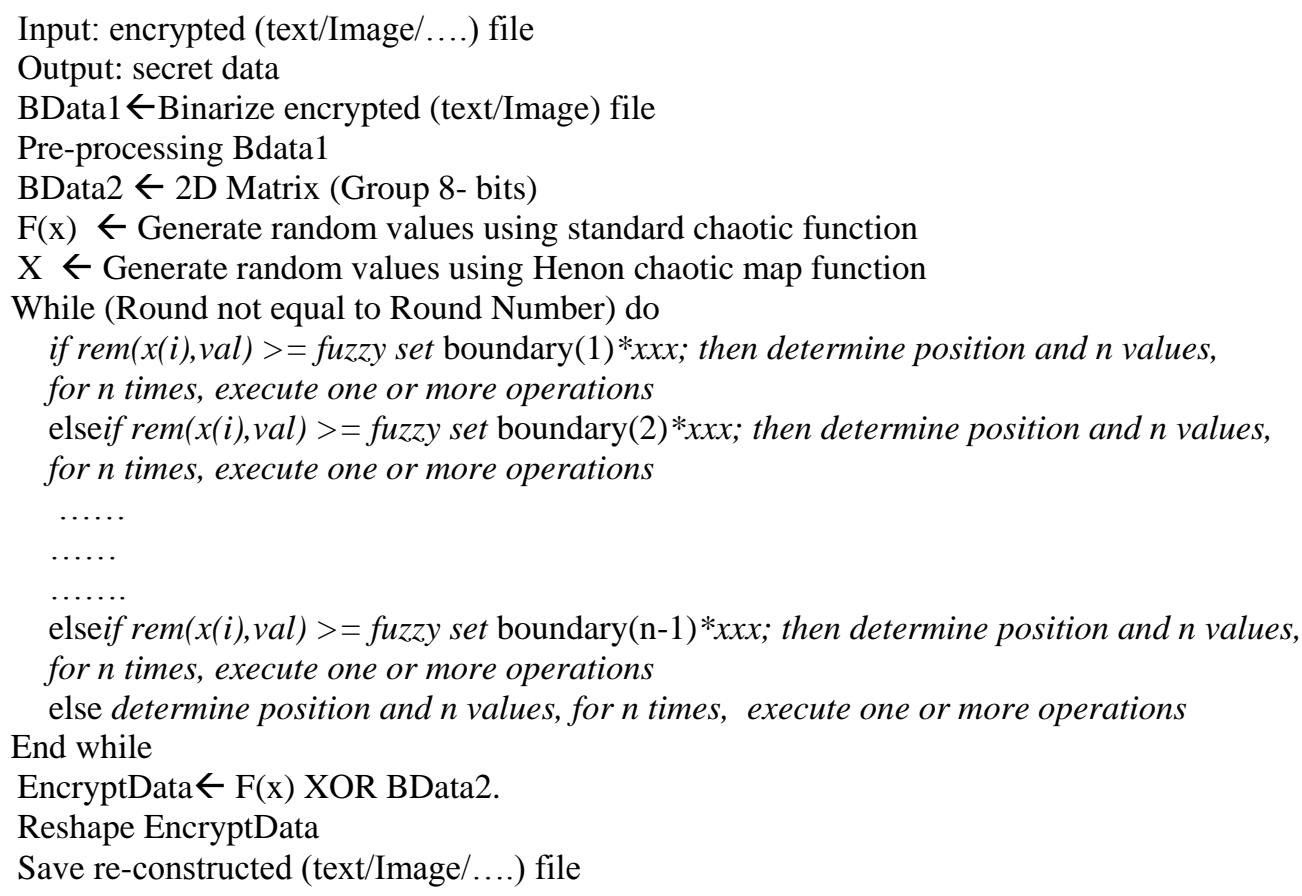

Fig.4. Pseudocode of Proposed Decoding C-GET

The Pseudocode of Encoding and Decoding secure CGET are shown in figure 3 and figure 4.

In the previous explanation of C-GET, the preprocessing stage depends on each type of data file and format. It is wide-ranging among to conversion to ASCII values, reading pixels of the image, separating components or frame and getting the properties of the video file as mentioned above to solve this problem, generalize C-GET technique. The generalization technique is composed of the same stages of C-GET but, the process of read data and the pre-processing stage are replaced with a simple read binary file command (fread) using 8-bit unsigned integer (uint8) and specified parameters that describe the format of the secret data. 


\section{IMPLEMENTATION AND EVALUATION RESULTS}

The C-GET is implemented using MATLAB 2012 on Windows 8.1 64-bit Operating system in AMD Athlon (tm) II X2 220 Processor, 2.80GHz and 4 GB RAM. We perform number of experiments to test and evaluate the effectiveness of the proposed technique and run it with different types of secret data. We use different sizes images, for example, to check the technique performance by testing encryption strength and original image recovery. The strength of encryption is been evaluated using two of the most common quantity; the number of pixel changing rate (NPCR) and the unified average changed intensity (UACI) [34-35]. Peak signal-to-noise ratio (PSNR) is used to test image quality.

In the first part, some experiments are carried out to prove the efficiency of the proposed C-GET with different formats of gray and color images. We choose some known test images and others as test images. First, read image.

In case of RGB image, separate firstly it to three components, then each component passes through the processes of C-GET to encrypt it. After encoding, gathering, reshape and save encrypted data in file. The original images and encrypted-images in image format are shown in Figure 5. The processing time for encrypting $256 \times 256$ gray image file is approximately 0.5 seconds/256 rounds. The processing time for encrypting $256 \times 256$ RGB image file is approximately 3.3 seconds/256 rounds. Some gray and true color images and their encrypted images format are shown in figure 5.

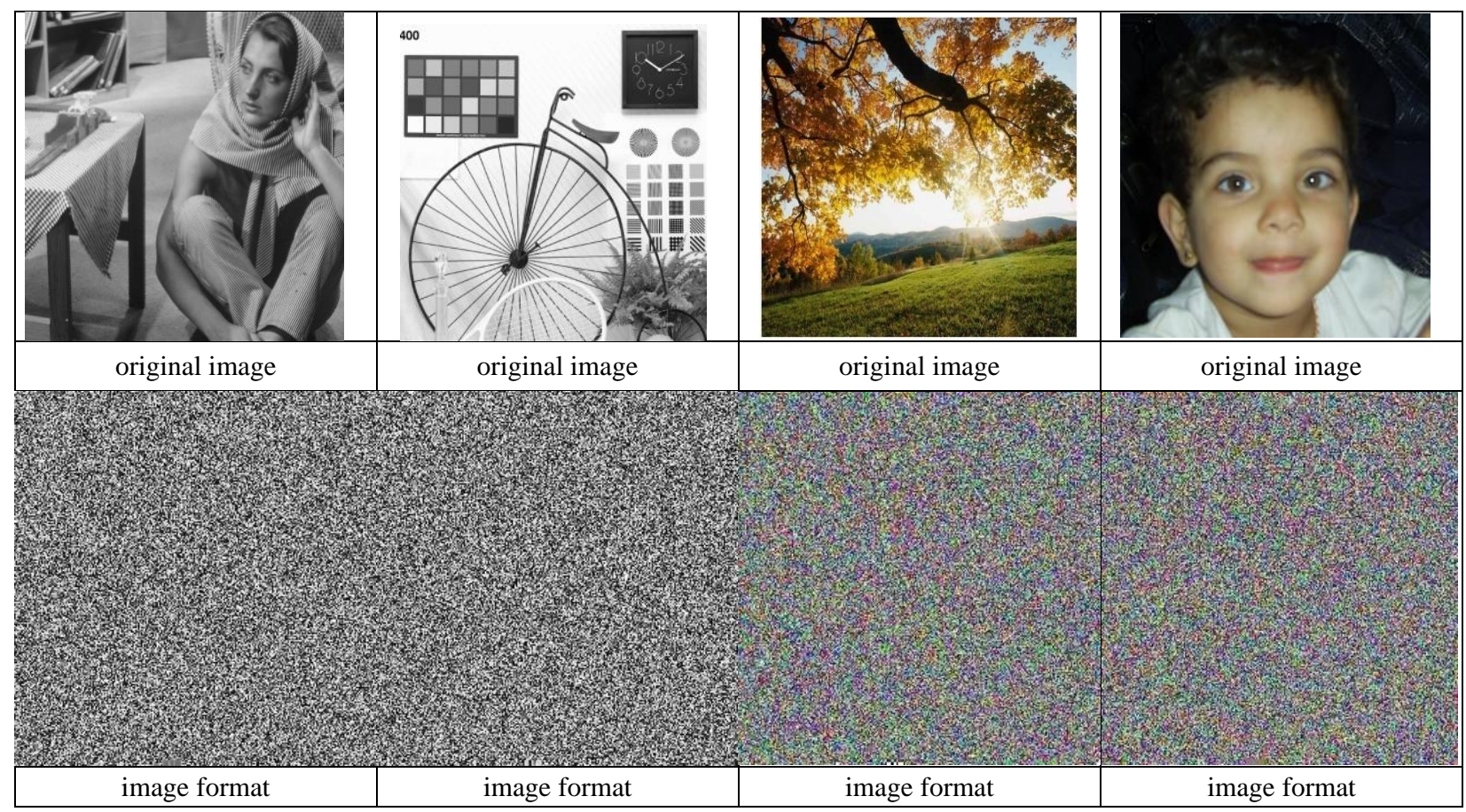

Fig.5. Original Images and Their Encrypted

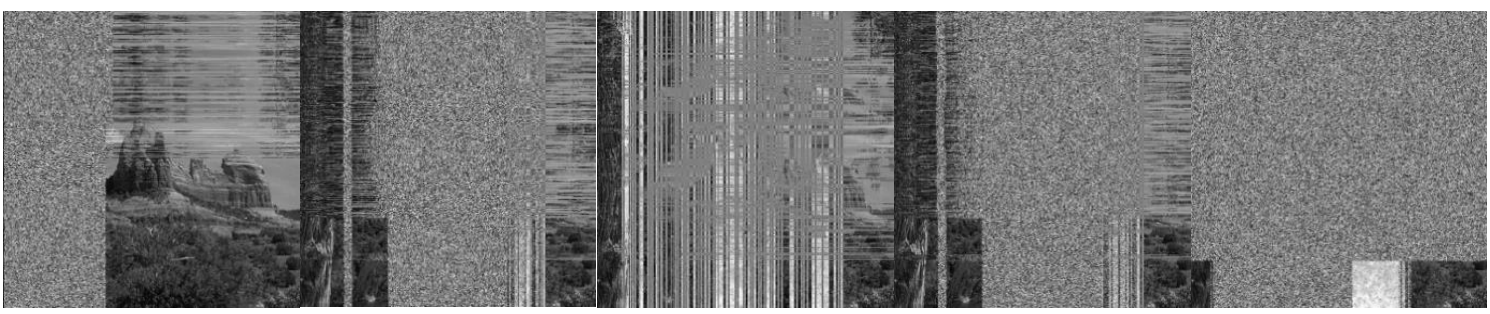

Fig.6. Encrypted Images with Uncover All Data with Fuzzy Rules

In case of insufficient representation, rules and the rules do not cover all data size; the encryption technique has a very low-security level and is not good technique. Some examples of encrypted data are shown in figure 6 due to using the encrypted technique with lack of rules and uncover all data with fuzzy rules.

\section{Histogram Analysis}

The histogram is a graphical representation that shows the occurrence of pixels intensity values. To prevent an attack, the obtained cipher image should give no indication about the original image. In the proposed approach, the obtained cipher image does not give any indication of the original image, whichis analyzed through histograms. A sampleof gray and RGB images, corresponding encrypted images and their histograms are shown in figure 7. 


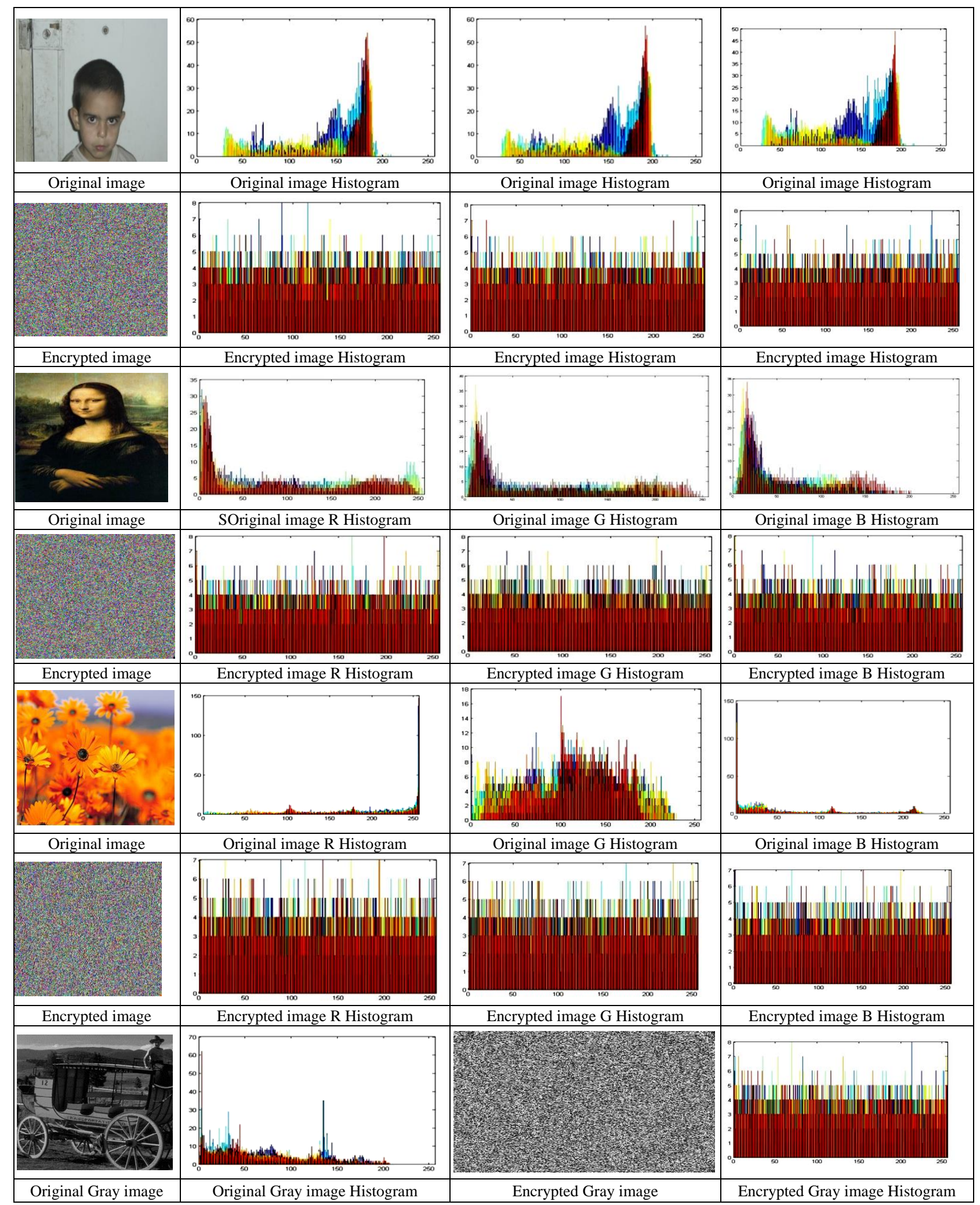

Fig.7. Original Image, Histogram of original image, Encrypted Image and Histogram of Encrypted Image

\section{Correlation Coefficient Analysis}

The correlation among neighboring pixels for the most part of the original images is high, while the correlation is a little between adjacent pixels in the encrypted image [36]. The following equationdefinescorrelation coefficient:

$$
\text { correlation coefficient }=\frac{\sum_{\mathrm{m}} \sum_{\mathrm{n}}\left(\mathrm{A}_{\mathrm{mn}}-\overline{\overline{\mathrm{A}}}\right)\left(\mathrm{B}_{\mathrm{mn}}-\overline{\overline{\mathrm{B}}}\right)}{\sqrt{\sum_{\mathrm{m}} \sum_{\mathrm{n}}\left(\mathrm{A}_{\mathrm{mn}}-\overline{\overline{\mathrm{A}}}\right)^{2}\left(\mathrm{~B}_{\mathrm{mn}}-\overline{\overline{\mathrm{B}}}\right)^{2}}}
$$

Where $\mathrm{A}, \mathrm{B}$ are matrices or vectors of the same size. $\overline{\bar{A}}=$ Average of (A) matrix elements, and $\overline{\bar{B}}=$ Average of (B) matrix elements. It is known that: the value of 
correlation coefficient is around zero at highly uncorrelated sequences. In addition, the correlation coefficient almost equal to one when there is strongly correlated sequences.

By using MATLAB function (corr2 (I, J)), the correlation coefficient equals value in range -0.0029 to 0.0059 . It is mean that the relation between the original and encrypted imagesis negligible and the encrypted imagedoesnot give any information to the attacker about the original image. This elimination correlation between pixels indicates efficient encryption technique.

\section{Entropy}

Entropy is a numerical measure of randomness or disorder in any system that uses to describe the texture of the input image. The following equation defines Entropy:

$$
\text { Entropy }=-\sum P \log _{2}(P)
$$

Where; P contains the histogram counts.

The entropy is the randomness or uncertainty measure in a secret data, as entropy approaches 8 , there is more randomness [37]. Entropy values of the original image and its correspondent encrypted image have been given in figure 8 . The obtained results provide evidence that our proposed technique has the ability against entropy attack.

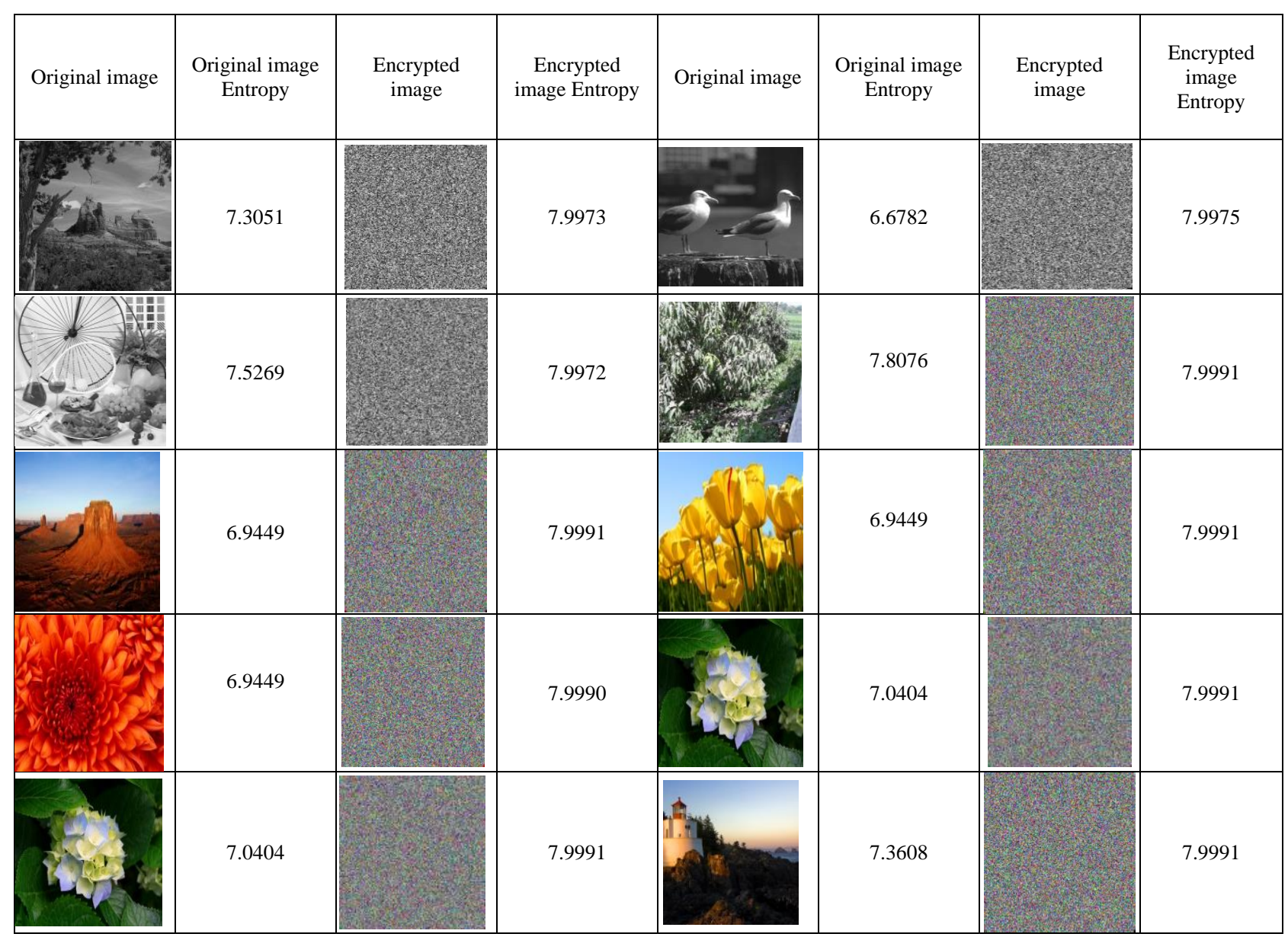

Fig.8. Entropy Analysis of Original Image and Its Correspondent Encrypted Image

\section{NPCR and UACI Analysis}

Two parameters number of pixel changing rate (NPCR) and unified average changed intensity (UACI) confirms the ability of proposed technique of resistance against differential attacks. NPCR is the change rate of pixels in the encrypted image when changing only one pixel of the original image and defined as follows:

$$
\operatorname{NPCR}(A, B)=\frac{1}{m \times n}\left(\sum_{i, j} \operatorname{sim}(i, j)\right) \times 100 \%
$$

And sim is represented by Equation:

$$
\operatorname{sim}(i, j)= \begin{cases}1 & \text { if } A(i, j)=B(i, j) \\ 0 & \text { if } A(i, j) \neq B(i, j)\end{cases}
$$

UACI measures the percentage differences between the original intensity and encrypted image intensity that using the following Equation:

$$
\operatorname{UACI}(A, B)=\frac{1}{m \times n}\left\{\sum_{i . j}\left|\frac{A(i, j)-B(i, j)}{\max \_ \text {pixel_value }}\right|\right\} \times 100 \%
$$

Where A, B are two corresponding the original and encrypted images to same original image, $\mathrm{m}$ and $\mathrm{n}$ are dimensions of A and B, and max_pixel_value denotes the 
largest value in the original image [34-35].

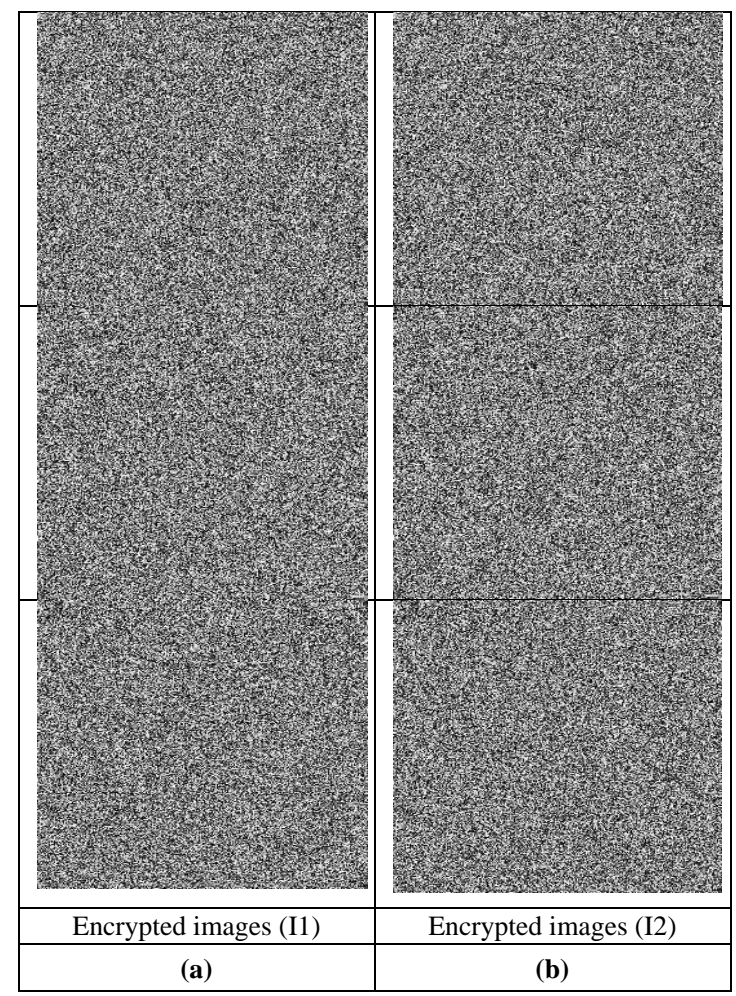

Fig.9. Two Encrypted Images Corresponding to Change 1 pixel in Original Image before Encryption

These parameters are used for testing the effect of changing of a single pixel in the original image on the encrypted image. They also used to test and verify its' resistance to the differential attacks [26, 29].

In this test, we randomly change the value of only one pixel from original image "I1". The obtained image is denoted by "I2", then encrypt both images (I1 and I2) using the proposed technique. Two encrypted images (A \& B) corresponding to "I1" and "I2", respectively are shown in figure $9(a, b)$. The average of NPCR and UACI are 0.0015 and 31.53931 respectively.

\section{Key Space Analysis}

The C-GET has large key space that making brute force attack is infeasible Because of using more than chaotic faction type. It's known that the chaotic system is sensitive to a tiny change in initial condition or parameters and fuzzy rules also have several different values that add space to key size and different combinations of them. So it resists the exhaustive of brute force attacks.

Thus, the C-GET makes larger statistical changes in the transmitted images. A cryptosystem is secure if it cannot be discovered even with full knowledge of the decryption algorithm. The gotten results prove that proposed technique can overcome many known and existsteganalytic attacks. Its output depends on initial parameters of chaotic map functions and types of fuzzy memberships, fuzzy rules, operation functions and sequence of functions execution that making a prediction of original secret data is very complex and an increase of computing time. It has a higher level of security against some existing attacks based on the multiple-operations, fuzzy-genetic operations and number of rounds. In general, it realizes better encryption.

\section{CONCLUSIONS}

In this paper, C-GET is designed and implemented. The C-GET enhances secured encryption technique based on multi-iterations, two types of chaotic map functions, fuzzy logic and genetic operations. It is also included mathematic operations, encryption and rotation increase encryption quality. The results show the resistance of the C-GET technique against different attacks based on several parameters, operations sequence of C-GET. The original and reconstructed images are identical. The encrypted-data is more randomness and has negligible correlation with original data so the cryptanalysis' possibilities for breaking the cipher are negligible. Furthermore, the technique has multilayer protection stages that achieve confidentiality, gives more security, effectiveness and robustness to data, and protects against detection.

In future work, we will be optimizing and standardizing C-GET and try to reduce the transmitted data size and the encryption time.

\section{REFERENCES}

[1] William Stallings, "Cryptography and Network Security: Principles and Practice", Prentice Hall, 2011.

[2] Keith M. Martin,” Everyday Cryptography Fundamental Principles and Applications", Oxford University Press Inc., New York, 2012.

[3] B. Schneier, Applied Cryptography, John Wiley \& Sons, New York, 1994.

[4] R. L. Rivest, A. Shamir and L. Adleman, "A Method for Obtaining Digital Signatures and Public-Key Cryptosystems," Communication ACM, Vol. 21, No. 2, pp. 120126, 1978.

[5] Charles P. Pfleeger, Shari Lawrence Pfleeger, Jonathan Margulies, "Security in Computing", FIFTH EDITION,Pearson Education, Inc., 2015.

[6] Janailin Warjri and Dr. E. George Dharma Prakash Raj," KED - A Symmetric Key Algorithm for Secured Information Exchange Using Modulo 69", I. J. Computer Network and Information Security, vol., 10, pp. 37-43, 2013.

[7] Sajasi, S.; Eftekhari Moghadam, A. M.,"A high quality image hiding scheme based upon noise visibility function and an optimal chaotic based encryption method", IEEE, AI \& Robotics and 5thRoboCup Iran Open International Symposium (RIOS), 2013.

[8] Subhajit Das, Satyendra Nath Mandal, Sunil Karforma, "Fuzzy-GA Combined Approach in Image Encryption" International Journal of Computer Sciences and Engineering (www.ijcseonline.org) Vol.-4(6), pp. 137-142, Aug 2016.

[9] Omar A. Dawood, Abdul Monem S. Rahma, Abdul Mohsen J. Abdul Hossen,"The New Block Cipher Design (Tigris Cipher)", IJCNIS, vol.7, no.12, pp. 10-18, 2015.

[10] Ahmed Y. Mahmoud, Alexander G. Chefranov, "A Hill Cipher Modification Based on Eigenvalues Extension with Dynamic Key Size HCM-EXDKS", IJCNIS, vol.6, 
no.5, pp.57-65, 2014.

[11] Hinal M. Mudia and Pallavi V. Chavan, "Fuzzy Logic Based Image Encryption for Confidential Data Transfer Using $(2,2)$ Secret Sharing Scheme", Procedia Computer Science 78, pp. $632-639,2016$.

[12] K. Ganesh Kumarand D. Arivazhagan" New Cryptography Algorithm with Fuzzy Logic for Effective Data Communication", Indian Journal of Science and Technology, Vol. 9(48), pp. 1-6, December 2016.

[13] Musheer Ahmad, Hamed D. Alsharari and Munazza Nizam, "Security Improvement of an Image Encryption Based on mPixel-Chaotic-Shuffle and Pixel-ChaoticDiffusion", arXiv: $1403.6626 \quad$ [cs.CR], 2014. (https://arxiv.org/ftp/arxiv/papers/1403/1403.6626.pdf)

[14] GAMIL R.S. QAID1, SANJAY N. TALBAR, "Encrypting Image by Using Fuzzy Logic Algorithm", International Journal of Image Processing and Vision Sciences (IJIPVS), Vol-2, Iss-1, pp. 25-29, 2013.

[15] Mani. K, Viswambari. M, "Enhancing the Security in Cryptosystems Based on Magic Rectangle", International Journal of Computer Network and Information Security, Vol.9, No.4, pp. 37-47, 2017.

[16] Naveed Ahmed Azam,"A Novel Fuzzy Encryption Technique Based on Multiple Right Translated AES Gray S-Boxes and Phase Embedding", Security and Communication Networks, pp. 1-9 Volume 2017.

[17] Michael François, Thomas Grosges, Dominique Barchiesi, Robert Erra, "Image Encryption Algorithm Based on a Chaotic Iterative Process", Applied Mathematics (http://www.SciRP.org /journal/am), 3, pp. 1910-1920, 2012.

[18] Jawad Ahmad and Fawad Ahmed, "Efficiency Analysis and Security Evaluation of Image Encryption Schemes", International Journal of Video \& Image Processing and Network Security IJVIPNS-IJENS Vol: 12 No. 04, pp. 1931, 2012.

[19] Noorul Hussain Ubaidur Rahman, Chithralekha Balamurugan and Rajapandian Mariappan,"A Novel DNA Computing based Encryption and Decryption Algorithm", Procedia Computer Science 46 pp. $463-475,2015$. (International Conference on Information and Communication Technologies (ICICT 2014)

[20] Fatma E. Ibrahim, M. I. Moussa and H. M. Abdalkader, "A Symmetric Encryption Algorithm based on DNA Computing", International Journal of Computer Applications (0975 - 8887) Volume 97- No.16, pp. 41-45, July 2014.

[21] Zhang, Q., Guo, L. and Wei, X., "Image encryption using DNA addition combining with chaotic maps", Mathematical and Computer Modelling 52, pp. 2028-2035, 2010.

[22] Hamdy M. Mousa, "DNA-Genetic Encryption Technique", I. J. Computer Network and Information Security, No. 7, pp. 1-9, 2016.

[23] Scharinger, J., "Fast encryption of image data using chaotic Kolmogorov flows", Journal of Electronic Imaging 7, pp. 318-325, 1998.

[24] Fridrich, J., "Symmetric ciphers based on twodimensional chaotic maps", International Journal of Bifurcation and Chaos 8, pp. 1259-1284, 1998.
[25] Chen G, Mao Y B, Chui C K., "A symmetric image encryption scheme based on 3D chaotic cat maps", Chaos, Solitons \& Fractals 21, pp. 749-761, 2004.

[26] Mao Y, Chen G, Lian S., "A novel fast image encryption scheme based on 3D chaotic Baker maps", International Journal of Bifurcation and Chaos 14, pp. 3613-3624, 2004.

[27] Liu H, Zhu Z, Jiang H, Wang B., “A Novel Image Encryption Algorithm Based on Improved 3D Chaotic Cat Map", The 9th IEEE International Conference for Young Computer Scientists, pp. 3016-3021, 2008.

[28] Huang, C. K., Nien, H. H., "Multi chaotic systems based pixel shuffle for image encryption", Optics Communications 282, pp. 2123-2127, 2009.

[29] Edward N. Lorenz, "Deterministic nonperiodic flow", in Journal of the Atmospheric Sciences, Vol. 20, pp.130 $141,1963$.

[30] Hénon M., "A two-dimensional mapping with a strange attractor", Communications in Mathematical Physics. 50(1), pp. 69-77, 1976.

[31] George J. Kllr and Bo Yuan, "Fuzzy Sets and Fuzzy Logic Theory and Applications", Prentice Hall PTR, New Jersey, 1995

[32] Timothy J. Ross, "Fuzzy Logic with Engineering Applications", John Wiley \& Sons Ltd, 2004.

[33] David A. Coley, "AN INTRODUCTION TO GENETIC ALGORITHMS FOR SCIENTISTS AND ENGINEERS", World Scientific Publishing Co. Pte. Ltd, 1999.

[34] Yue Wu, Joseph P. Noonan, and Sos Agaian, "NPCR and UACI Randomness Tests for Image Encryption", Cyber Journals: Multidisciplinary Journals in Science and Technology, Journal of Selected Areas in Telecommunications (JSAT), April Edition, 2011.

[35] Nisar Ahmed, Hafiz Muhammad Shahzad Asif, Gulshan Saleem,"A Benchmark for Performance Evaluation and Security Assessment of Image Encryption Schemes", International Journal of Computer Network and Information Security(IJCNIS), Vol.8, No.12, pp.18-29, 2016.

[36] Moore, D.S., "The basic practice of statistics", W. H. Freeman and Company, New York, 2009.

[37] Robert M. Gray, Entropy and information theory, Springer-Verlag New York, Inc., New York, NY, 1990.

\section{Authors' Profiles}

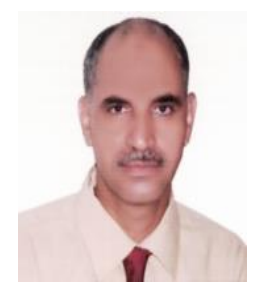

Hamdy M. Mousa received the B.S. and M.S. in Electronic Engineering and Automatic control and measurements from Menoufia University, Faculty of Electronic Engineering in 1991 and 2002, respectively and received his $\mathrm{PhD}$ in Automatic control and measurements Engineering (Artificial intelligent) from Menoufia University, Faculty of Electronic in 2007. His research interest includes intelligent systems, Natural Language Processing, privacy, Security, embedded systems, GSP applications, intelligent agent, Robotics.

How to cite this paper: Hamdy M. Mousa,"Chaotic Genetic-fuzzy Encryption Technique", International Journal of Computer Network and Information Security(IJCNIS), Vol.10, No.4, pp.10-19, 2018.DOI: 10.5815/ijcnis.2018.04.02 